\title{
Risk of Coronary Heart Events Based on Rose Angina Questionnaire and ECG Besides Diabetes and Other Metabolic Risk Factors: Results of a 10-Year Follow-up in Tehran Lipid and Glucose Study
}

\author{
Mohammad Ali Mansournia, ${ }_{1}$ Kourosh Holakouie-Naieni, ${ }^{1}$ Noushin Fahimfar, ${ }^{1}$ Amir \\ Almasi-Hashiani, ${ }^{1}$ Zahra Cheraghi, ${ }^{2}$ Erfan Ayubi, ${ }^{1}$ Farzad Hadaegh, ${ }^{3}$ Fatemeh Eskandari, ${ }^{3}$ Fereidoun \\ Azizi, ${ }^{4}$ and Davood Khalilii ${ }^{3,5},{ }^{*}$ \\ ${ }^{1}$ Department of Epidemiology and Biostatistics, School of Public Health, Tehran University of Medical Sciences, Tehran, Iran \\ ${ }^{2}$ Department of Epidemiology, School of Public Health, Hamadan University of Medical Sciences, Hamadan, Iran \\ ${ }^{3}$ Prevention of Metabolic Disorders Research Center, Research Institute for Endocrine Sciences, Shahid Beheshti University of Medical Sciences, Tehran, Iran \\ ${ }^{4}$ Endocrine Research Center, Research Institute for Endocrine Sciences, Shahid Beheshti University of Medical Sciences, Tehran, Iran \\ ${ }^{5}$ Department of Biostatistics and Epidemiology, Research Institute for Endocrine Sciences, Shahid Beheshti University of Medical Sciences, Tehran, Iran \\ "Corresponding author: Davood Khalili, Prevention of Metabolic Disorders Research Center, Research Institute for Endocrine Sciences, Shahid Beheshti University of Medical \\ Sciences, Tehran, Iran. Tel: +98-2122432500, Fax: +98-2122416264, E-mail: dkhalili@endocrine.ac.ir
}

Received 2016 October 01; Revised 2017 February 27; Accepted 2017 April 09.

\begin{abstract}
Background: High-risk individuals for CHD could be diagnosed by some non-invasive and low-priced techniques such as Minnesota ECG coding and rose angina questionnaire (RQ).

Objectives: The present study aimed at determining the risk of incident CHD according to ECG and RQ besides diabetes and other metabolic risk factors in our population.

Methods: Participants comprised of 5431 individuals aged $\geq 30$ years within the framework of Tehran lipid and glucose study. Based on their status on history of CHD, ECG, and RQ at baseline, all participants were classified to 5 following groups: (1) History Rose ECG (the reference group); (2) History Rose ${ }^{+} \mathrm{ECG}^{-}$; (3) History Rose $\mathrm{ECG}^{+}$; (4) History Rose $\mathrm{ECG}^{+}$; and (5) History ${ }^{+}$. We used Cox regression model to find the role of ECG and RQ on CHD, independent of other risk factors.

Results: Overall, 562 CHD events were detected during the median of 10.3 years follow-up. CHD incidence rates were 55.9 and 9.09 cases per 1000 person-year for participants with and without history of CHD, respectively. Hazard ratios (HRs) (95\% CIs) were 4.11 (3.27 - 5.11) for History + and $2.18(1.63-2.90), 1.92(1.47-2.51)$, and 2.48 (1.46 - 4.20) for History Rose ${ }^{+}$ECG $^{-}$, History Rose ECG ${ }^{+}$, and History Rose $^{+} \mathrm{ECG}^{+}$, respectively. RQ and ECG had the same HRs as high as those for hypertension and hypercholesterolemia; however, diabetes showed statistically and clinically more effects on CVD than RQ and ECG.

Conclusions: RQ in general and, ECG especially in asymptomatic patients, were good predictors for CHD events in both Iranian males and females; however, their predictive powers were lower than that of diabetes.

Keywords: Coronary Heart Disease, Population Based Cohort, Rose Questionnaire, Electrocardiography, Diabetes, Hypertension, Hypercholesterolemia
\end{abstract}

\section{Background}

Urbanization phenomenon with industrialization has led to epidemiological transition from communicable diseases to chronic diseases $(1,2)$. Noncommunicable diseases are the main cause of death worldwide (1-3). Cardiovascular disease (CVD) is considered as the main cause of death in most countries with some well-known metabolic risk factors like diabetes, hypertension, and hypercholesterolemia (4). CVD is also the first and most common cause of death in Iran (5). Recently, in a study performed by Talaei et al. on an Iranian population in Isfahan, the mortality rate of CVD was reported to be 331 and 203 per 100000 person-years in males and females, respectively (6). CVDs were also found as a cause of more than $40 \%$ of deaths in Tehran (7), and the incidence rate of CHD has been 6.5 and 11.9 per 1000 person-years in females and males of this capital city, respectively (8). On the other hand, the prevalence of CHD was $10.7 \%$, 6\%, and $11.8 \%$ based on Rose Angina Questionnaire (RQ), self-report, and electrocardiogram (ECG) in this city, respectively (9). However, the incidence of recurrent CVD has not yet been reported in Iran.

Early detection of CHD plays an important role in early intervention and reduces future risk of serious disease. There are simple and low cost methods in populationbased studies for assessing high risk individuals such as 
RQ, ie, a standardized instrument for measuring typical angina in population surveys (10), and Minnesota ECG coding , a more objective measure for CHD events in populations $(11,12)$.

To understand the usefulness and effectiveness of each screening test, the 4 following key points should be noted: (1) the cost of the screening test; (2) the frequency of undesirable detected outcome; (3) the relationship between abundance and survival of diagnosed patients; and (4) the ability to reduce adverse outcomes (morbidity and mortality) by the information obtained from the screening test (13).

\section{Objectives}

In the present study, we aimed at assessing the roles of ECG and RQ in predicting new CHD events, independent of other cardiovascular risk factors, and assessing the incidence rate of recurrent $\mathrm{CHD}$ among people with a positive history. Moreover, we aimed at comparing the predictive power of these measurements with that of diabetes and other metabolic risk factors. More detailed information helps predict CHD events precisely and improves quality of care services (14).

\section{Methods}

\subsection{Study Population}

This study was conducted in the framework of Tehran lipid and glucose study (TLGS), which is a population-based cohort study in Tehran with the aim of determining the incidence and prevalence of noncommunicable diseases and their risk factors. This study has been described in details before $(15,16)$. There were 8071 individuals aged 30 years or older in the first phase of TLGS, of whom 5976 participants completed ECG and RQ. Among the 5976 participants, 545 individuals were excluded due to lack of follow-up, and the remained 5431 participants were followed until March 20, 2010 with a median follow-up of 10.3 years (Figure 1). Informed consent was signed by the all participants, and the study protocol was approved by the ethics committee of research institute for endocrine sciences at Shahid Beheshti University of Medical Sciences.

\subsection{Exposure}

The participants were categorized into 3 groups according to the Minnesota code and Whitehall criteria: (1) probable CHD (codes of 1.1.1 through 1.1.7, and 1.2.1 through 1.2.8); (2) possible CHD (codes of 1.3.1 through 1.3.6; 4.1.1 through $4.4 ; 5.1$ through 5.3 or 7.1.1 through 7.1.2); (3) and non-CHD (ECGs that had none of these criteria) (17). We defined ECG-positive CHD as probable or possible CHD at baseline. In the study, the Persian version of RQ was used as it is a reliable tool for detecting angina pectoris in Iranian population (18).

At baseline, the participants were classified into 5 categories based on self-reported history of CHD, ECG, and RQ results: 1, History Rose-ECG: negative CHD history, ECG

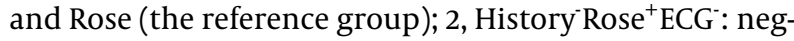
ative CHD history, negative ECG, and positive Rose; 3, History ${ }^{-}{ }^{-} \mathrm{ECG}^{+}$: negative CHD history, negative Rose, and positive ECG; 4, History ${ }^{-} \mathrm{Rose}^{+} \mathrm{ECG}^{+}$: Negative CHD history, positive ECG and positive Rose; and 5, History ${ }^{+}$: positive self-reported CHD history.

\subsection{Outcome}

The outcome was defined as the occurrence of CHD or death from CHD. The participants were censored at the time of their last follow-up, death due to any other causes or at the end of study (March 20, 2010), and whichever came first.

In brief, each participant was under continuous followup for any CHD event leading to hospitalization or death, confirmed by an outcome committee. Details of the CHD outcome data have been published previously(8). The CHD outcome was comparable with ICD-10 rubric I20-I25.

\subsection{Potential Confounders}

At the baseline visit (i.e, 1999 - 2001), data were collected using interview, physical examination, and laboratory measurements. Using pretested questionnaires, trained interviewers interviewed the participants. Information on age, gender, medical history of CHD, smoking habit, and family history of CVD were collected. A blood sample was taken after 12 to 14 hours of overnight fasting. The measurements of fasting plasma glucose (FPG), 2h plasma glucose (2hPG), systolic and diastolic blood pressure (SBP and DBP), HDL cholesterol (HDL-C), and total cholesterol (TC) have been described in detail elsewhere (19). Participants were classified as diabetic if they reported the use of glucose lowering medications, FPG $\geq 7.0$ $\mathrm{mmol} / \mathrm{l}(126 \mathrm{mg} / \mathrm{dl})$ or $2 \mathrm{~h}-\mathrm{PG} \geq 11.1 \mathrm{mmol} / \mathrm{l}(200 \mathrm{mg} / \mathrm{dl})$. Hypertension was defined as systolic/diastolic blood pressure equal or higher than $140 / 90 \mathrm{~mm} \mathrm{Hg}$ or using hypertension medication; and hypercholesterolemia was defined as serum cholesterol $\geq 6.21 \mathrm{mmol} / \mathrm{l}(240 \mathrm{mg} / \mathrm{dl})$ or using lipid drugs.

\subsection{Statistical Methods}

Baseline characteristics of the participants were presented as mean (SD) or frequency (percentage). KaplanMeier survival curves were constructed for the 5 study 


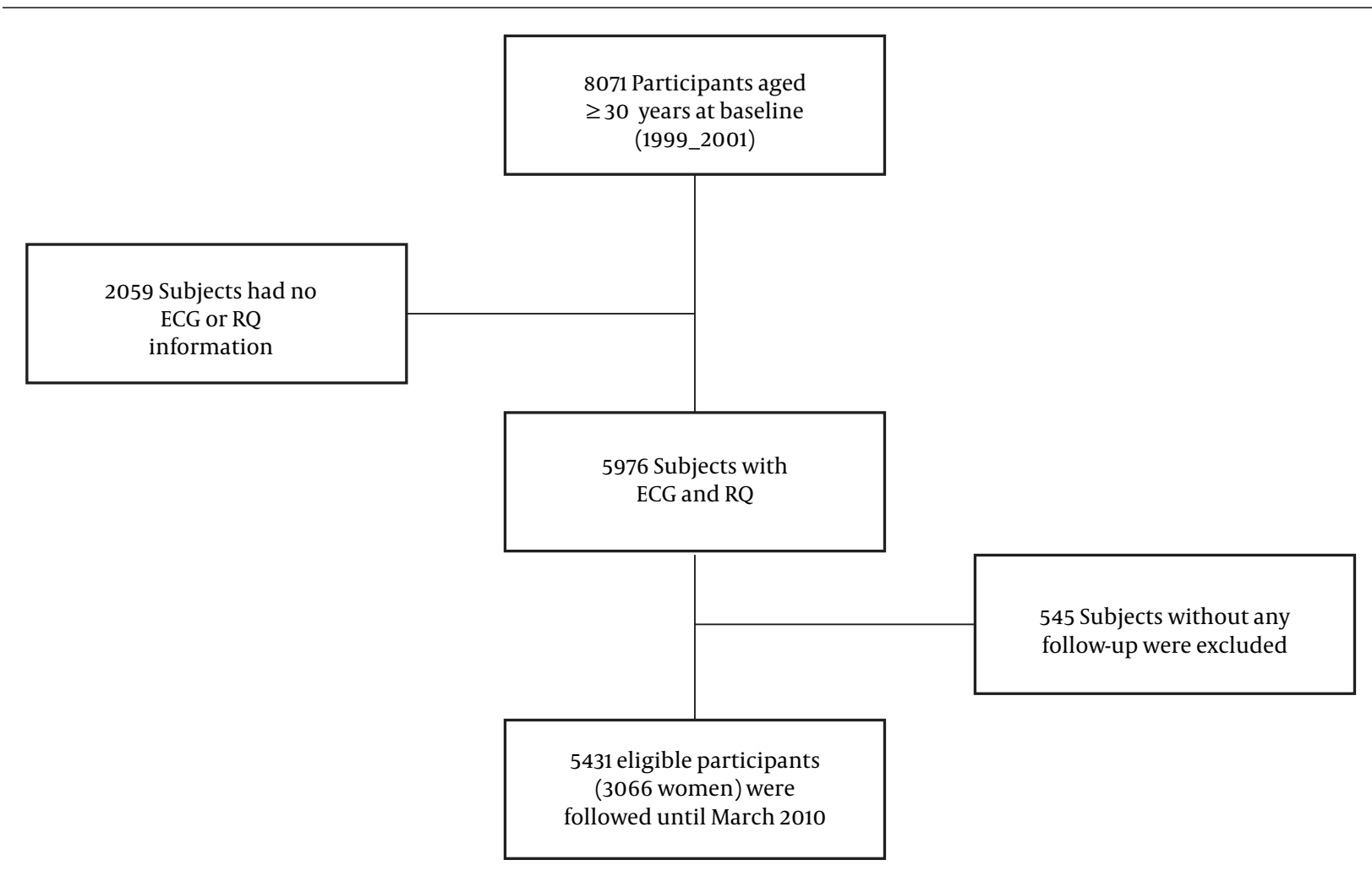

Figure 1. Study population flowchart

groups and compared using the log-rank test. The hazard ratios with 95\% confidence intervals (95\% CIs) in groups (2-5) relative to the reference (History-Rose-ECG-) were estimated using the Cox proportional-hazards regression model. Multivariable Cox regression model was used to estimate the hazard ratios ( $95 \%$ CIs) adjusted for the potential confounders mentioned above. To finely adjust for the confounding effect of age, the time origin was set to date of birth, making time-since-birth analysis time. The Cox proportional hazards assumption was visually assessed by log$\log$ plot, ie, the plot of - $\log -\log (\mathrm{S}(\mathrm{t})$ ) curves for levels of CHD history against $\log (\mathrm{t})$. The interaction between gender and study groups was tested in the analysis and because there was no significant interaction, a pooled regression analysis over gender was conducted to obtain more efficient estimates. Adjusted comparisons between the study groups in pairs were conducted using the multivariable Cox regression models. Using Wald test, the HRs of metabolic risk factors (hypertension, hypercholesterolemia, and diabetes) were compared to the HRs of the study groups. All statistical analyses were conducted using Stata software, Version 11 (Stata Corp, College Station, TX, USA).

\section{Results}

Generally, 2575 (43.1\%) of the 5976 participants were male, of whom 167 (6.5\%) had positive history of CHD. Positive history of CHD was detected in 157 (4.6\%) of females. Baseline characteristics of the study participants in each level of CHD history are presented in Table 1.

A total of 562 CHD events occurred during 50856 person-year of follow-up, of which 320 events were in males (21 626 years of follow-up). As presented in Table 2, CHD incidence rate among all participants was 11.05 cases per 1000 person-year. Incidence rates of CHD in males with and without history of CHD were 62.87 (95\% CI: 49.39 80.03) and 12.35 (95\% CI:10.92 - 13.96) cases per 1000 personyear, respectively. These figures were 49.26 (95\% CI: 37.63 64.48) and 6.71 (95\% CI: 5.82 - 7.74) in females.

Kaplan-Meier survival curves for the 5 categories are demonstrated in Figure 2. Although a significant difference was shown in general $(\mathrm{P}<0.001)$, the Kaplan Meier survival curves for History Rose $^{-} \mathrm{ECG}^{+}$and History Rose $^{+}$ECG $^{-}$were the same. The approximately parallel curves in log-log plot suggest that the Cox proportional hazards assumption was satisfied for the CHD history (Figure 3). The results of the multivariable Cox 
Table 1. Baseline Characteristics by CHD History ${ }^{\mathrm{a}}$ of 5976 Participants in Tehran Lipid and Glucose Study

\begin{tabular}{|c|c|c|c|c|}
\hline \multicolumn{2}{|c|}{ Variables } & \multirow{2}{*}{$\begin{array}{c}\text { With History of CHD }(\mathbf{n}=\mathbf{3 2 4}) \\
157(48.5)\end{array}$} & \multirow{2}{*}{$\begin{array}{c}\text { Without History of } \mathbf{C H D}(\mathbf{n}=\mathbf{5 6 5 2}) \\
3244(57.4)\end{array}$} & \multirow{2}{*}{$\begin{array}{r}\text { P Value } \\
0.002\end{array}$} \\
\hline Gender & Female & & & \\
\hline Diabetes & Yes & $89(27.5)$ & $568(10.05)$ & 0.001 \\
\hline Hypertension & Yes & $211(65.1)$ & $1443(25.5)$ & 0.001 \\
\hline Hypercholesterolemia & Yes & $146(45)$ & $1655(29.3)$ & 0.001 \\
\hline Cigarette smoking & Yes & $108(33.3)$ & $1333(23.6)$ & 0.001 \\
\hline \multirow{2}{*}{ BMI } & $25-30$ & $149(46)$ & $2458(43.5)$ & \multirow{2}{*}{0.021} \\
\hline & $\geq 30$ & $102(31.4)$ & $1529(27)$ & \\
\hline HDL & $<40$ & $173(53.4)$ & $2782(49.2)$ & 0.14 \\
\hline Age (years); Mean (SD) & & $60.3(9.5)$ & $47.1(12.06)$ & 0.001 \\
\hline
\end{tabular}

${ }^{\mathrm{a}}$ No.(\%), except where otherwise indicated.

Table 2. Incidence Rate of CHD by the Group Variable of the Study Separately in Male and Female Participants

\begin{tabular}{|c|c|c|c|c|c|}
\hline \multirow{2}{*}{\multicolumn{2}{|c|}{ Variables }} & \multirow[t]{2}{*}{ Number of New Cases } & \multirow[t]{2}{*}{ Incidence Rate (IR) Per 1000} & \multicolumn{2}{|c|}{ 95\% CI for IR } \\
\hline & & & & Lower & Upper \\
\hline \multicolumn{6}{|l|}{ Male } \\
\hline & History Rose ECG & 186 & 10.4 & 9.0 & 12.0 \\
\hline & History Rose ${ }^{+}$ECG & 27 & 26.0 & 17.8 & 37.8 \\
\hline & History Rose $\mathrm{ECG}^{+}$ & 33 & 23.5 & 16.7 & 33.1 \\
\hline & History Rose $^{+} \mathrm{ECG}^{+}$ & 8 & 44.6 & 22.3 & 89.1 \\
\hline & $\mathrm{CHD}^{+}$ & 66 & 62.9 & 49.4 & 80.0 \\
\hline \multicolumn{6}{|c|}{ Female } \\
\hline & History Rose ECG & 119 & 5.1 & 4.3 & 6.1 \\
\hline & History Rose ${ }^{+} \mathrm{ECG}^{-}$ & 29 & 12.6 & 8.8 & 18.2 \\
\hline & History Rose $\mathrm{ECG}^{+}$ & 34 & 15.2 & 10.8 & 21.2 \\
\hline & History Rose ${ }^{+} \mathrm{ECG}^{+}$ & 7 & 24.4 & 11.6 & 51.2 \\
\hline & $\mathrm{CHD}^{+}$ & 53 & 49.3 & 37.6 & 64.5 \\
\hline \multicolumn{6}{|c|}{ Both sex } \\
\hline & History Rose ECG & 305 & 7.4 & 6.6 & 8.2 \\
\hline & History Rose ${ }^{+} \mathrm{ECG}^{-}$ & 56 & 16.7 & 12.9 & 21.8 \\
\hline & History Rose ${ }^{-} \mathrm{ECG}^{+}$ & 67 & 18.3 & 14.4 & 23.3 \\
\hline & History Rose ${ }^{+} \mathrm{ECG}^{+}$ & 15 & 32.1 & 19.3 & 53.3 \\
\hline & $\mathrm{CHD}^{+}$ & 119 & 55.9 & 46.7 & 67.0 \\
\hline Total & & 526 & 11.05 & 10.17 & 12.0 \\
\hline
\end{tabular}

regression model are presented in Table 3. Adjusting for potential confounders, the hazard ratio of new CHD in participants with CHD history was 4.11 (95\% CI: 3.27 - 5.11) times of the reference group. Compared to the reference group, the hazard ratios in participants with positive Rose, positive ECG, and both were 2.18 (95\% CI: 1.63 - 2.90), 1.92
(95\% CI: 1.47 - 2.51), and 2.48 (95\% CI: 1.46 - 4.20), respectively (Table 3). Furthermore, except for BMI and HDL, all confounders had a significant relationship with CHD events.

The HRs between study groups and CHD events were presented separately in males and females (Table 4). Com- 
Table 3. Multivariable Cox Regression Model for CHD

\begin{tabular}{|c|c|c|}
\hline \multirow[t]{2}{*}{ Variables } & \multicolumn{2}{|l|}{ Total $(n=5431)$} \\
\hline & HR (\%95 CI) & P Value \\
\hline \multicolumn{3}{|l|}{ Groups (History-Rose-ECG- as reference) } \\
\hline History ${ }^{-10} \mathrm{e}^{+} \mathrm{ECG}^{-}$ & $2.18(1.63-2.90)$ & $<0.001$ \\
\hline History Rose $\mathrm{ECG}^{+}$ & $1.92(1.47-2.51)$ & $<0.001$ \\
\hline History Rose $^{+} \mathrm{ECG}^{+}$ & $2.48(1.46-4.20)$ & 0.001 \\
\hline $\mathrm{CHD}^{+}$ & $4.11(3.27-5.11)$ & $<0.001$ \\
\hline Gender (male vs. female) & $1.91(1.56-2.34)$ & $<0.001$ \\
\hline Familial history of CVD (yes vs. no) & $1.37(1.12-1.67)$ & 0.002 \\
\hline Diabetes (yes vs. no) & $3.04(2.52-3.67)$ & $<0.001$ \\
\hline Hypercholesterolemia (yes vs. no) & $1.64(1.37-1.96)$ & $<0.001$ \\
\hline Hypertension (yes vs. no) & $2.10(1.75-2.52)$ & $<0.001$ \\
\hline Cigarette smoking (yes vs. no) & $1.42(1.16-1.73)$ & $<0.001$ \\
\hline HDL $(\geq 40$ vs. $<40)$ & $0.85(0.72-1.02)$ & 0.085 \\
\hline \multicolumn{3}{|l|}{ Body mass index ( $<25$ as reference $)$} \\
\hline $25-30$ & $1.09(0.88-1.35)$ & 0.42 \\
\hline$\geq 30$ & $1.06(0.83-1.36)$ & 0.59 \\
\hline
\end{tabular}

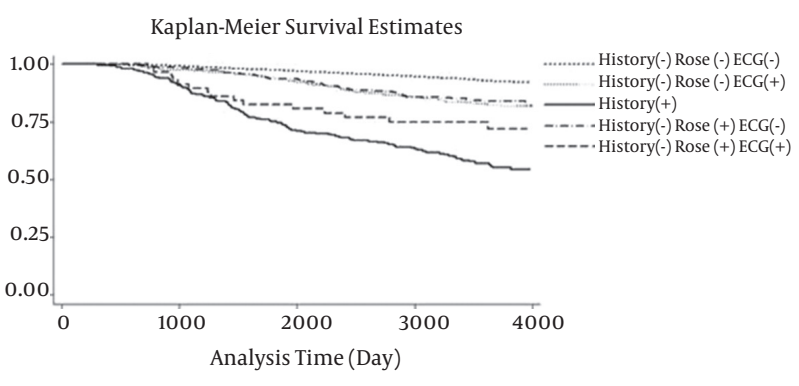

Figure 2. Kaplan-Meier Survival Curves by history, RQ and ECG

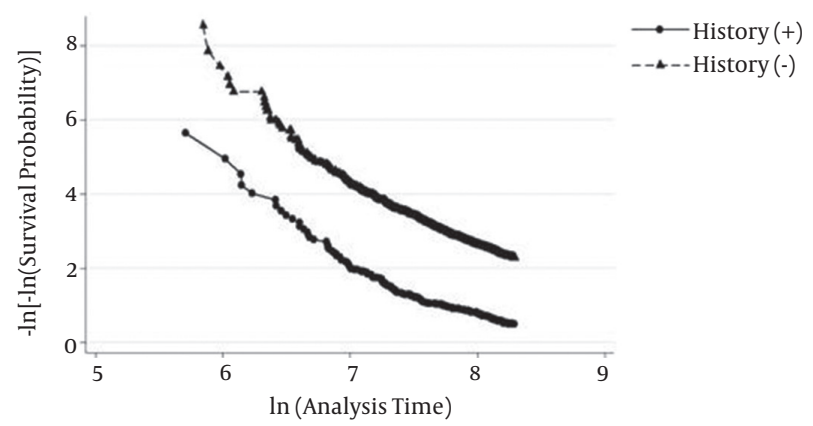

Figure 3. Log-Log Plot for Checking the Proportional Hazards Assumption pared to the reference group, the hazard ratios for all other study groups in both genders were both clinically important and statistically significant at $5 \%$ level, but with no important differences between genders $(\mathrm{P}$ for interaction $=$ 0.65).

Compared to the History Rose $^{+} \mathrm{ECG}^{-}$group, there were no significant differences in History ${ }^{-}{ }^{-}{ }^{-} \mathrm{ECG}^{+}$(HR: 0.88; 95\% CI: $0.62-1.26$ ) and History ${ }^{-}{ }^{-} \mathrm{Ne}^{+} \mathrm{ECG}^{+}$(HR: 1.14, 95\% CI: 0.64 - 2.02) groups. Considering History Rose $^{-} \mathrm{ECG}^{+}$as the reference, no significant difference was detected in History ${ }^{-}{ }^{+}{ }^{+} \mathrm{ECG}^{+}$group (HR: 1.29; 95\% CI: 0.74 - 2.27).

In a separate analysis, all problematic participants with no history of CHD (including History Rose ${ }^{+} E C G$, History ${ }^{-}$Rose ${ }^{-} \mathrm{EG}^{+}$and History ${ }^{-}$Rose $^{+} \mathrm{ECG}^{+}$) were compared with History+ patients. The significant differences were highlighted (HR: 3.34; 95\% CI: 2.69 - 4.13).

With respect to the metabolic risk factors, the hazard ratios for History ${ }^{-}{ }^{+}{ }^{+}{ }^{+} \mathrm{ECG}^{-}$and History ${ }^{-}$Rose ${ }^{-} \mathrm{ECG}^{+}$were statistically as same as the HRs of hypertension and hypercholesterolemia.

Diabetes showed a statistically significant difference with History Rose ${ }^{-} \mathrm{EGG}^{+}$participants (HR of 3.04 vs. 1.92 , P $=0.007$ ) and borderline difference with History ${ }^{-}$Rose $^{+} \mathrm{ECG}^{-}$ group (HR of 3.04 vs. 2.18, $\mathrm{P}=0.056$ ). 
Table 4. Multivariable Cox Regression Model for CHD Based on Gender Groups

\begin{tabular}{|c|c|c|c|c|}
\hline \multirow[b]{2}{*}{ Variables } & \multicolumn{2}{|c|}{ Females $(n=3066)$} & \multicolumn{2}{|c|}{ Males $(n=2365)$} \\
\hline & $\operatorname{HR}(95 \% \mathrm{CI})$ & PValue & $\mathrm{HR}(95 \% \mathrm{CI})$ & PValue \\
\hline \multicolumn{5}{|c|}{ Groups (History Rose ECG as reference) } \\
\hline History Rose ${ }^{+}$ECG & $2.06(1.37-3.10)$ & $<0.001$ & $2.37(1.58-3.55)$ & $<0.001$ \\
\hline History-Rose-ECG+ & $2.27(1.55-3.34)$ & $<0.001$ & $1.66(1.14-2.41)$ & 0.008 \\
\hline History-Rose+ECG+ & $2.85(1.32-6.13)$ & 0.007 & $2.23(1.09-4.56)$ & 0.028 \\
\hline $\mathrm{CHD}+$ & $4.57(3.27-6.40)$ & $<0.001$ & $3.80(2.84-5.08)$ & $<0.001$ \\
\hline
\end{tabular}

\section{Discussion}

We compared the participants according to the presence of symptom (Rose Angina) or sign (ECG) or both/none of them at baseline. The risk of recurrent CHD among people with history of CHD was also evaluated. Our study with more follow-up time, confirmed the Khalili et al. findings that positive Rose Angina predicts the CHD events among Iranian people even in the presence of other cardiovascular risk factors, but not as much as diabetes (20).

Sensitivity and specificity of the Rose questionnaire may be different between countries (21). We found that Rose questionnaire as a good screening tool is appropriate to predict the risk and add information on undiagnosed $\mathrm{CHD}$ in both genders ( $\mathrm{HR}=2.37$ in males and 2.06 in females). ECG has also been introduced as a strong predictive tool in some studies previously $(22,23)$.

The prediction power of ECG could not been found by Khalili et al. and the Rose-ECG ${ }^{+}$groups had HR: 1.36 with a nonsignificant effect compared to baseline. Because no interaction was found between gender and Rose/ECG groups, we pooled both sexes for analysis. The analyses with more power showed the strong role of ECG to predict CHD in patients with silent ischemia.

However, we could define increased risk of CHD events among $\operatorname{Rose}^{+} \mathrm{ECG}^{+}$population in parallel with the findings by Hemingway et al. (24). The limited sample size in this group might have caused the nonsignificant result through loss of power in the previous study.

We found no significant difference in the risk of CHD between Rose $^{+} \mathrm{ECG}^{-}$and Rose $\mathrm{ECG}^{+}$in both genders. These findings revealed the same risk in symptomatic patients (Rose $\mathrm{ECG}^{-}$) and the asymptomatic patients with positive sign (Rose $\mathrm{ECG}^{+}$), especially in females.

More than $62 \%$ of males and $49 \%$ of females with selfreported history of CHD had experienced recurrent CHD during the follow- up. Considering participants without any history/symptom and sign of CHD as the reference, we found that the risk of new CHD in males and females with positive self-reported history were more than 3.8 and 4.57 folds, respectively.

In ARIC study, a population-based cohort of people aged 45 to 64 years, 766 CHD patients (189 females were followed for recurrent CVD events. During a mean of 8.7 years of follow-up, 313 acute CVD occurred, resulting in a recurrent CVD event rate of 47 per 1000 person-years (41 in females and 49 in males). The percentage of participants who had an acute CVD event by 10 years of follow-up was $38.7 \%$ for females and $45.1 \%$ for males (25).

The incidence of recurrent cardiovascular outcomes among patients with Type 2 diabetes was calculated by Giorda et al. They followed 2788 patients with diabetes aged 40 to 97 years with CVD at enrollment. During 4 years of follow-up, the incidence of a recurrent CVD was 72.7 per 1000 person-years (95\% CI: 58.3 - 87.1) and 32.5 per 1000 person-years (95\% CI: 21.2 - 43.7) in males and females, respectively (26). Moreover, in another similar cohort study, with a median follow-up of 4.1 years, that was conducted by Heijden et al., the incidence rate of recurrent events per 100 person-years was $12.5(8.5-17.6)$ in individuals with Type 2 diabetes (27). In Cha et al. study patients had recurrent episodes of CVD, with an incidence rate of 75.6 per 1000 patient-years (28).

Diabetic patients are more prone to have recurrent CVDs, and known diabetic patients demonstrated a CHD risk similar to nondiabetic patients with a prior CHD in both genders (29).

Increased risk of subsequent CVD morbidity and mortality is related to traditional risk factors, geographic location, and lack of treatment (30). Our study showed that hypertension and hypercholesterolemia had the same HRs as high as those for patients with positive findings in RQ or ECG. Moreover, it was found that diabetes has statistically and clinically important effects on CVD outcomes more than hypertension and hypercholesterolemia. For clinicians, prevention of new CVD and its recurrence in patients with previous CVD is an overwhelming task. We found that even participants with positive self-reported history had 
significant differences in either symptoms or signs compared to other problematic patients with negative history. Because the absolute risk is greater for this group, considering the high incidence rate of recurrent CHD in our population, this issue should seriously be considered in our country. Moreover, preventing first CHD in our high incident country is so important. The risk factors for recurrent CVDs are generally assumed tto be as same as the first ones, so controlling for the occurrence and proper interventions are necessary and results in preventing new and recurrent CVDs. There is ample evidence showing that a multiplicity of drug treatments and behavioral changes can reduce morbidity and mortality for those with existing CHD such as stopping smoking, maintaining a healthy diet, physical activity, and taking appropriate drug treatment (31).

Even with more analysis, we could not detect any statistically significant differences at 5\% level for the risk of incident CHD between prevalent cases of CHD at baseline and those with no history of CHD who had both positive Rose Angina and abnormal ECG (HR: 1.66; 95\% CI: 0.96 - 2.84; P = $0.06)$; and this might be a result of low power due to small sample size in history Rose $^{+} \mathrm{ECG}^{+}$group. To our knowledge, no study was available which compared the risk of CHD in these 2 groups.

\subsection{Study Strengths and Weaknesses}

Our study had some restrictions, which should be kept in mind for better interpretation. First, these results cannot expand easily to the entire population, especially rural individuals. Secondly, both major and minor ECG abnormalities were considered as ECG abnormalities in our analysis. Because minor ECG abnormalities (ie, ST depression, T-wave items, small Q, or QS wave) may be related to other medical situations (ie, hyperventilation, anxiety, food ingestion, and change in posture), we might have attenuated the value of ECG changes in predicting incident CHD.

\subsection{Conclusions}

The rate of recurrent CHD in positive self-reported history of CHD in our community is high and it should be considered more precisely in practice. We found that each RQ and ECG has its own role in predicting CHD events. Rose questionnaire can be considered as a simple and helpful clinical screening tool among Iranian population with high prevalence of CHD even in the presence of normal ECG. However, ECG should be measured in the risk assessment of asymptomatic individuals. The predictive powers of these measurements were as same as that of hypercholesterolemia and hypertension, but lower than that of diabetes.

\section{Acknowledgments}

We appreciate the TLGS committee members and those who participated in this study.

\section{Footnotes}

Authors' Contribution: Mohammad Ali Mansournia, Kourosh Holakouie-Naieni, Fereidoun Azizi and Davood Khalili conceptualized the study design; Noushin Fahimfar, Davood Khalili, and Mohammad Ali Mansournia coordinated and supervised the study; Farzad Hadaegh, Fatemeh Eskandari, and Fereidoun Azizi were responsible for data collection, editing, and management; Zahra Cheraghi, Amir Almasi-Hashiani, Erfan Ayubi, Noushin Fahimfar, and Mohammad Ali Mansournia supervised sample collection, and data analysis and interpretation; Zahra Cheraghi, Amir Almasi-Hashiani, Erfan Ayubi, and Noushin Fahimfar drafted the article; Mohammad Ali Mansournia and Davood Khalili critically revised the manuscript for important intellectual content and provided final approval of the version to be published; All authors reviewed and approved the final draft before publication.

Financial Disclosure: None declared.

Funding/Support: The TLGS has been supported by grant No. 121 from the national research council of Iran; however, the present stud, which was conducted in the framework of the TLGS, had no specific financial support.

\section{References}

1. Yusuf S, Reddy S, Ounpuu S, Anand S. Global burden of cardiovascular diseases: part I: general considerations, the epidemiologic transition, risk factors, and impact of urbanization. Circulation. 2001;104(22):2746-53. [PubMed: 11723030].

2. Global status report on noncommunicable diseases. World Health Organization; 2011.

3. Keil U. The worldwide WHO MONICA Project: results and perspectives. Suppl 1. ; 2005. p. S38e45.

4. Nichols M, Ownsend N, Luengo-Fernandez R, Leal J, Gray A. European cardiovascular disease statistics. Europ Society Cardiol. 2012.

5. Esteghamati A, Meysamie A, Khalilzadeh O, Rashidi A, Haghazali M, Asgari F, et al. Third national Surveillance of Risk Factors of NonCommunicable Diseases (SuRFNCD-2007) in Iran: methods and results on prevalence of diabetes, hypertension, obesity, central obesity, and dyslipidemia. BMC Public Health. 2009;9:167. doi: 10.1186/14712458-9-167. [PubMed: 19480675].

6. Talaei M, Sarrafzadegan N, Sarrafzadegan N, Sadeghi M, Oveisgharan S. Incidence of cardiovascular diseases in an Iranian population: the isfahan cohort study. Arch Iran Med. 2013;16(3).

7. Khalili D, Mosavi-Jarrahi A, Eskandari F, Mousavi-Jarrahi Y, Hadaegh F, Mohagheghi M, et al. Evaluation of cause of deaths' validity using outcome measures from a prospective, population based cohort study in Tehran, Iran. PLoS One. 2012;7(2):31427. doi: 10.1371/journal.pone.0031427. [PubMed: 22355365]. 
8. Khalili D, Sheikholeslami FH, Bakhtiyari M, Azizi F, Momenan AA, Hadaegh F. The incidence of coronary heart disease and the population attributable fraction of its risk factors in Tehran: a 10year population-based cohort study. PLoS One. 2014;9(8):105804. doi: 10.1371/journal.pone.0105804. [PubMed: 25162590].

9. Hadaegh F, Harati H, Ghanbarian A, Azizi F. Prevalence of coronary heart disease among Tehran adults: Tehran Lipid and Glucose Study. East Mediterr Health J. 2009;15(1):157-66. [PubMed: 19469439].

10. Rose GA. The diagnosis of ischaemic heart pain and intermittent claudication in field surveys. Bull World Health Organ. 1962;27(64):58.

11. Keys A, Taylor H, Blackburn H. Coronary heart disease among Minnesota Business and Professional men followed fifteen years. Circulation. 1963;28(381):95.

12. Moon JC, Fisher NG, McKenna WJ, Pennell DJ. Detection of apical hypertrophic cardiomyopathy by cardiovascular magnetic resonance in patients with non-diagnostic echocardiography. Heart. 2004;90(6):645-9. [PubMed: 15145868].

13. Grimes DA, Schulz KF. Uses and abuses of screening tests. Lancet. 2002;359(9309):881-4. doi: 10.1016/S0140-6736(02)07948-5. [PubMed: 11897304].

14. Kubo M, Kiyohara Y, Kato I, Tanizaki Y, Arima H, Tanaka K, et al. Trends in the incidence, mortality, and survival rate of cardiovascular disease in a Japanese community: the Hisayama study. Stroke. 2003;34(10):2349-54. doi: 10.1161/01.STR.0000090348.52943.A2. [PubMed: 12958323].

15. Azizi F, Ghanbarian A, Momenan AA, Hadaegh F, Mirmiran P, Hedayati $M$, et al. Prevention of non-communicable disease in a population in nutrition transition: Tehran Lipid and Glucose Study phase II. Trials. 2009;10:5. doi:10.1186/1745-6215-10-5. [PubMed:19166627].

16. Azizi F, Rahmani M, Emami H, Mirmiran P, Hajipour R, Madjid M, et al. Cardiovascular risk factors in an Iranian urban population: Tehran lipid and glucose study (phase 1). Soz Praventivmed. 2002;47(6):40826. [PubMed: 12643001]

17. Ford ES, Giles WH, Croft JB. Prevalence of nonfatal coronary heart disease among American adults. American heart J. 2000;139(3):371-7.

18. Najafi-Ghezeljeh T, Ekman I, Nikravesh MY, Emami A. Adaptation and validation of the Iranian version of Angina Pectoris characteristics questionnaire. Int J Nurs Pract. 2008;14(6):470-6. doi: 10.1111/j.1440172X.2008.00722.x. [PubMed: 19126076].

19. Zabetian A, Hadaegh F, Azizi F. Prevalence of metabolic syndrome in Iranian adult population, concordance between the IDF with the ATPIII and the WHO definitions. Diabetes Res Clin Pract. 2007;77(2):251-7. doi: 10.1016/j.diabres.2006.12.001. [PubMed: 17234299].

20. Khalili D, Hadaegh F, Fahimfar N, Shafiee G, Sheikholeslami F, Ghanbarian $\mathrm{A}$, et al. Does an electrocardiogram add predictive value to the rose angina questionnaire for future coronary heart disease? 10-year follow-up in a Middle East population. J Epidemiol Community Health. 2012;66(12):1104-9. doi: 10.1136/jech-2011-200242. [PubMed: 22544919].

21. Rose G. Variability of angina. Some implications for epidemiology. $\mathrm{Br}$ JPrev Soc Med. 1968;22(1):12-5. [PubMed: 5636970].

22. Denes P, Larson JC, Lloyd-Jones DM, Prineas RJ, Greenland P. Major and minor ECG abnormalities in asymptomatic women and risk of cardiovascular events and mortality. JAMA. 2007;297(9):978-85. doi 10.1001/jama.297.9.978. [PubMed: 17341712].

23. Daviglus ML, Liao Y, Greenland P, Dyer AR, Liu K, Xie X, et al. Association of nonspecific minor ST-T abnormalities with cardiovascular mortality: the Chicago Western Electric Study. JAMA. 1999;281(6):5306. [PubMed: 10022109].

24. Hemingway H, Shipley M, Britton A, Page M, Macfarlane P, Marmot M Prognosis of angina with and without a diagnosis: 11 year follow up in the Whitehall II prospective cohort study. BMJ. 2003;327(7420):895. doi: 10.1136/bmj.327.7420.895. [PubMed:14563744].

25. Wattanakit K, Folsom AR, Chambless LE, Nieto FJ. Risk factors for cardiovascular event recurrence in the Atherosclerosis Risk in Communities (ARIC) study. Am Heart J. 2005;149(4):606-12. doi: 10.1016/j.ahj.2004.07.019. [PubMed: 15990741].

26. Giorda CB, Avogaro A, Maggini M, Lombardo F, Mannucci E, Turco S et al. Recurrence of cardiovascular events in patients with type 2 diabetes: epidemiology and risk factors. Diabetes Care. 2008;31(11):21549. doi: 10.2337/dc08-1013. [PubMed: 18782902].

27. van der Heijden AA, Van't Riet E, Bot SD, Cannegieter SC, Stehouwer $\mathrm{CD}$, Baan CA, et al. Risk of a recurrent cardiovascular event in individuals with type 2 diabetes or intermediate hyperglycemia: the Hoorn Study. Diabetes Care. 2013;36(11):3498-502. doi: 10.2337/dc122691. [PubMed: 23877981].

28. Cha SA, Yun JS, Lim TS, Min K, Song KH, Yoo KD, et al. Diabetic Cardiovascular Autonomic Neuropathy Predicts Recurrent Cardiovascular Diseases in Patients with Type 2 Diabetes. PLoS One. 2016;11(10):0164807. doi:10.1371/journal.pone.0164807. [PubMed: 27741306].

29. Hadaegh F, Fahimfar N, Khalili D, Sheikholeslami F, Azizi F. New and known type 2 diabetes as coronary heart disease equivalent: results from 7.6 year follow up in a Middle East population. Cardiovasc Diabetol. 2010;9:84. doi: 10.1186/1475-2840-9-84. [PubMed: 21129219].

30. Wilson PW, D’Agostino RS, Bhatt DL, Eagle K, Pencina MJ, Smith SC, et al. An international model to predict recurrent cardiovascular disease. Am J Med. 2012;125(7):695-703 e1. doi: 10.1016/j.amjmed.2012.01.014. [PubMed: 22727237].

31. Bradley F, Cupples ME. Reducing the risk of recurrent coronary heart disease. We know a bit more about what doesn't work. BMJ. 1999;318(7197):1499-500. [PubMed: 10355981]. 